\title{
What Makes a Good Story - The Use and Acceptance of Storytelling in Business Intelligence
}

\author{
Saskia Ramm \\ Chemnitz University of Technology \\ saskia.ramm@,wirtschaft.tu-chemnitz.de \\ Barbara Dinter \\ Chemnitz University of Technology \\ barbara.dinter@wirtschaft.tu-chemnitz.de
}

\begin{abstract}
In the age of big data and analytics, the constantly growing complexity of information requires its suitable visualization. As an increasingly popular visualization technique, storytelling supports the successful discovery, presentation, and communication. However, a scientific discussion about the role of storytelling in Business Intelligence (BI) is still missing. Therefore, we consider it as beneficial to investigate this quite young phenomenon and its characteristics in more detail. In the paper we present a morphological box for storytelling in BI based on the results of an extensive literature review. In addition, we were interested to what extent BI users utilize and accept the storytelling concept. We have answered this research question by analyzing the use and acceptance of the storytelling feature in BI tools by adapting the Unified Theory of Acceptance and Use of Technology (UTAUT).
\end{abstract}

\section{Introduction}

Stories and narratives have always been used by humans to transmit information, experiences, ideas, and cultural values, as a well-told story provides not only more information but also makes it easier to understand for most people [15]. This type of communication is also of increasing interest to data analysts, who analyze data to extract information and knowledge and thus to allow decision-making [18].

Many years ago research has shown that the brain can process and retain visual information much more effectively than written or spoken information [7]. In practice, wrong or misinterpreted data analyses can result in serious consequences, such as expensive wrong decisions. Therefore, storytelling, a long-established method of presenting information, which allows us as human beings to gain insights and knowledge and creates wisdom [24], exhibits a suitable and valuable means in the age of big data and analytics.

\author{
Eva-Maria Kopf \\ Chemnitz University of Technology \\ e.kopf@gmx.de \\ Sarah Hönigsberg \\ Chemnitz University of Technology \\ sarah.hoenigsberg@,wirtschaft.tu-chemnitz.de
}

Especially in light of the current worldwide Covid-19 pandemic, we see that its dynamics and the devastating effects are simply not understood by many people. This shows the great danger of wrong or missing understanding of data, which can be overcome by storytelling to a certain extent.

Thereby, storytelling supports the analysis of data as well as the communication and presentation of the results by creating a story [12]. Stories can therefore be used to provide further information on the data and the corresponding context. This helps, for example, to present the results of decisions. Large amounts of data and information can be transmitted more efficiently and in an easily understandable manner [46]. Herschel and Clements [18] point out that (data) storytelling is a topic in the field of data visualization, which represents a structured approach to communicating relevant results from data analysis by combining data, visualization, and narratives.

In the field of Business Intelligence (BI) storytelling is already implemented as a feature in most tools [18, 29]. Moreover, according to a study by BARC about the most important BI trends, storytelling is one of the top trend topics in 2020 [1]. Therefore, this practicerelevant concept should also be addressed in scientific research. So far, rather few publications examine storytelling in BI, e. g. $[12,16,33]$ and its importance as a trend $[18,42]$. A systematic and scientific analysis of the topic is - to the best of our knowledge - still missing. Consequently, we see the need to focus on this important phenomenon. With our research we would like to contribute to the analysis and conceptualization of storytelling in $\mathrm{BI}$ as a field that is relevant for practice as well as for academia.

Thus, the paper addresses the following research questions (RQ): (1) How can the concept of storytelling in BI be characterized and (2) To which extent is the storytelling feature in BI tools used and accepted in practice? On the one hand, this paper aims to provide a deeper understanding of storytelling in BI. On the other hand, we are particularly interested in the actual use and 
acceptance of the storytelling feature in BI tools. In order to address this research gap, we conducted a systematic literature review about storytelling in BI and used the results for systematizing the phenomenon by means of a morphological box [48]. Moreover, we considered the user perspective and developed a model to measure the use and acceptance of the storytelling feature in BI tools empirically. To this end, in accordance with the Unified Theory of Acceptance and Use of Technology (UTAUT), a survey was conducted to empirically evaluate the model by Structural Equation Modeling (SEM). It aimed at identifying the determinants of the behavioral intention to use the storytelling feature in BI tools. In the following, we understand by users of storytelling in BI both the creators of stories, who use storytelling features to create a story, as well as the audience, whom the stories generated in this way are communicated to.

The paper at hand is structured as follows: First, we give an overview of the foundations of storytelling in general and in BI in particular before presenting the literature review and the resulting morphological box. Subsequently, we describe the research model and method of the empirical analysis. Afterwards, the results of the study are presented. Finally, we discuss our findings, show limitations of the paper, and provide an outlook for our future work.

\section{Foundations}

The main purpose of $\mathrm{BI}$ is to provide companies on all organizational levels with decision-relevant information [9]. It includes processes and systems, which assist the collection, processing, storage, analysis, and presentation of data in a suitable form [38]. Data visualizations with graphical elements (e. g. tables or diagrams) support data analysis and presentation [42] and are typically provided in the tool features reporting, dashboards, or storytelling.

The concept storytelling allows visualizations to illustrate information effectively as well as intuitively and aims to communicate the complexity of results [15, 46]. According to Kosara and Mackinlay [20], the purpose of storytelling is not only the visualization and communication of information to support decisionmaking, but also the facilitation of data analysis. Storytelling includes narrative structures, which is why research often calls it "Narrative Visualization" or "Visual Narratives" [35]. In the context of data visualization, researchers also use various terms, for example "Data Storytelling", "Data-driven Storytelling", "Data Stories", and "Visual Storytelling".

Lee et al. [22] present a systematic approach for storytelling with the key phases of data exploration, creating a story, and telling the story. The first phase includes data exploration and analysis activities in order to generate data excerpts as the foundation of the story [33]. A story can be created by compiling such excerpts in a storyline [16]. The last phase of telling a story requires the creation of story material by building a presentation and communicating the final story to the target audience $[16,33]$. The audience response and feedback about the perceived story can affect the (future) storytelling process [22]. Hence, the phases of the process do not have to be progressed in a fixed order and each should to be adapted on the target audience [22]. Furthermore, the process is influenced by other external factors such as the setting and medium [22]. These factors are discussed in more detail in the following section.

\section{Literature Review of Storytelling in BI}

We conducted a literature review according to the well-established methodology by Webster and Watson [43] in order to investigate the state-of-the-art of storytelling in BI. For this purpose we have selected the terms "Storytelling", "Narrative Visualization", "Business Intelligence" AND "Storytelling", "Visualization" AND "Storytelling", "Business Intelligence" AND "Narrative Visualization", "Business Intelligence" AND "Data Stories" as search terms. With the aim of providing high-quality data, we searched a number of international databases for highranking and/or domain-specific journal articles and conference proceedings. Therefore, the search process included the following electronic databases and search engines which we have selected based on their relevance for our research topic: ACM Library, AIS eLibrary, and IEEE Explore. Subsequently, we conducted a control search via Google Scholar. In addition, we included the domain-specific journals Decision Support Systems (DSS) and International Journal of Business Intelligence Research (IJBIR). The problem formulation and literature search was followed by a literature evaluation and analysis [43]. Reviewing titles and abstracts of the papers resulted in the first corpus of 33 relevant articles. We enhanced the output by a backward and forward search so that finally 43 relevant papers could be identified. In order to ensure a rigor and systematic review process, the analysis of the relevant papers was carried out independently by several researchers.

Due to the rather young research topic, it is not surprising that the majority of the papers has been published since 2010 and that related research has increased quite significantly over the last ten years. By analyzing the relevant articles, we identified eight dimensions that describe the phenomenon of storytelling in BI. The results are summarized in a morphological box in Table 1 . We will briefly discuss 
the dimensions (highlighted in bold font) and their attributes in the following.

A total of six application domains could be found, whereby most papers deal with storytelling in BI in the field of education and teaching. In many cases, storytelling is considered as a supporting medium in terms of both learning and teaching and thus for knowledge management as well as for transfer, e. g. [4, $11,26,27,32,41]$. As storytelling has its origins in journalism, there are also various research efforts in this area [31, 35, 36, 47]. Storytelling in BI is also suitable in science, as it makes scientific findings more easily accessible to the public. For example, first applications are already used in science museums and at NASA [25]. Further domains for storytelling in BI are business/organization [3, 8, 23], public administration [28], and social media [5, 44].

The dimension integration describes whether a standalone system is used for storytelling in BI or if it is implemented as a feature in a BI tool. While the literature review on the one hand shows that separate systems have been developed for storytelling in BI [10, 21], Pribisalić et al. [29] and also other authors (e. g. $[12,16,33,42])$ on the other hand point out that storytelling is an implemented feature in almost all BI tools.

Drawing on the paper by Elias et al. [12], we differentiate functions needed for the storytelling feature in BI in two dimensions, one referring to the functions for creating a story and the other to the relevant functions for the audience. Of course, selected functions, such as highlighting or annotating, can be assigned to both the creators and the audience of the story. Ultimately, the recipients of the story benefit from the functions that were used in the story creation. Therefore, we classified the functions by considering who uses the respective function primarily. We found the following functions for creators in the literature: automated storytelling [39], fluid transition, structure, reports, and reusable templates [12, 15, 37]. Further functions mainly focus on the visualization of data: interactive visualizations, highlighting, coloring, annotating, and explanations in text as well as in audio $[12,15,37]$. On the contrary, we have identified the following relevant functions for the audience of the story: playback, navigation, and zooming [12, 15, 37]. The dimension medium encompasses common forms of presentation and visualization of data, varying in information content and interactivity, as they can be found in the literature, e. g. [12, 22, 35, 37]. We could identify the media text, narration, table, chart, image, animation, video, audio, and interactive element. The medium dimension is related to the dimension 'functions for creators', as it represents the result of the applied functions to a certain extent. Furthermore, the appropriate medium has to be chosen in regard to the storytelling context [22].

The audience participation indicates if the audience has the possibility to take part in the story interactively (yes) or not (no).

In accordance with Lee et al. [22], we differentiate further the various roles of users and capture them in the dimension roles as data analysts, scripters, editors, moderators, or audiences. These different roles are involved in storytelling in BI in various ways. The data analyst examines the data, which forms the basis of the story. While the scripter creates the story based on the selected data extracts, the editor prepares the material [22]. Finally, the presenter is responsible for the presentation of the story to the audience. Obviously, there may be overlaps and one person may play several roles. In addition, further roles in the BI context could also be considered, such as administrators or database developers. However, we concentrate on deriving the dimensions and attributes from literature specifically for storytelling in BI.

Table 1. Morphological box for storytelling in BI

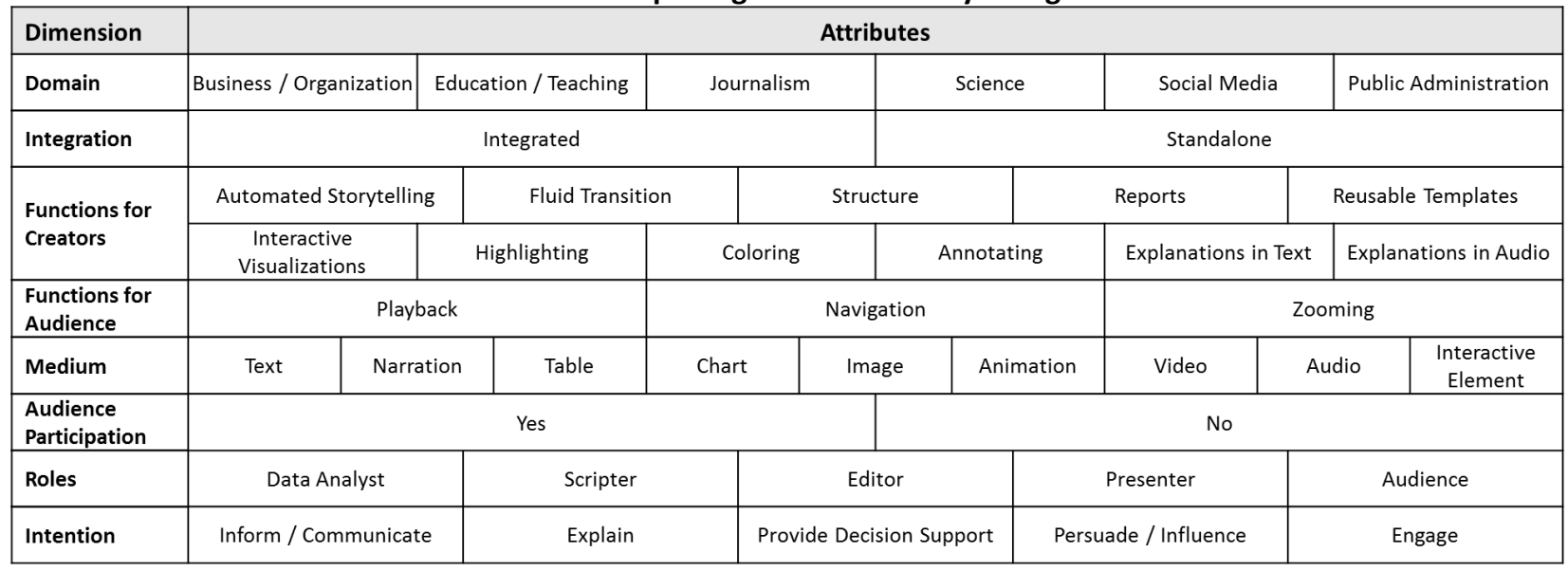


The last dimension considers the intention of why storytelling is used in BI. Thus, it refers to the key objectives of storytelling in BI. By compiling previous work, e.g. [2, 13, 15, 16, 18, 20, 42], we have identified the following attributes: inform/communicate, explain, provide decision support, persuade/influence, and engage.

In addition, we have compared our findings derived from the literature with the state of the art in practice. For example, we have analyzed selected BI tools in order to determine whether our insights correspond to storytelling features in BI tools. To this end, we selected four BI tools from the category leaders in Gartner's "Magic Quadrant for Analytics and Business Intelligence Platforms" [19]. The evaluation results are not in the focus of this paper, but we could confirm that functions derived from research have already been implemented in popular BI tools to a very large extent.

While the morphological box can serve as a starting point for the conceptualization of storytelling in BI, we would also like to investigate the actual use and acceptance of the storytelling feature in BI tools in the next Section.

\section{Empirical Investigation of Storytelling in BI Tools}

As already pointed out, storytelling can help to improve the cognitive processing of complex information [24] from data analysis in various scenarios. Consequently, users of BI tools can benefit from the storytelling feature, and therefore should increasingly accept it. Therefore, we investigated the use and acceptance of the storytelling feature in BI tools by developing a research model based on a popular acceptance model to investigate the topic from the user's perspective. Thus, we want to gain insights into the factors that contribute to the use and acceptance of the storytelling feature in BI tools in particular. Based on the understanding of these factors, additional design guidelines can be derived, whose consideration can have a positive impact on the successful use of the storytelling feature in BI tools.

The first subsection defines the research variables and hypotheses to be tested, followed by the presentation of the research design in Section 4.2.

\subsection{Research Model and Hypotheses}

Acceptance models can be used to identify the factors influencing the acceptance of technology and the individual intention of the person using a technology [45]. However, there is a large number of models in the scientific literature for measuring the acceptance of IT, which according to Williams et al. [45] leads to the challenge for researchers to choose the right theory for their research projects. Venkatesh et al. [40] addressed this problem in the development of the UTAUT acceptance model by integrating eight empirically tested theories. Thus, compared to other models, the UTAUT model achieved a large $\mathrm{R}^{2}\left(\mathrm{R}^{2}=0,70\right) \quad[40,45]$. Moreover, the adaptability of the model has already been tested in various scenarios [45]. Therefore, we have chosen the UTAUT model in its origin for our study.

The UTAUT model contains four core determinants of behavioral intention (BeIn) and use behavior (UB): performance expectancy (PE), effort expectancy (EE), social influence (SI), and facilitating conditions (FC). We have transferred the latent variables into our research context of the storytelling feature in BI tools and defined them according to Venkatesh at al. [40] as follows: Use behavior is the actual use of storytelling; behavioral intention, the willingness of the BI user to use storytelling and indicator for the acceptance; performance expectancy, the degree to which a BI user believes that using storytelling will help him or her to attain gains in job performance; effort expectancy, the ease of using storytelling; social influence, the degree to which an individual perceives that important others believe he or she should use storytelling; facilitating conditions, organizational and technical infrastructure and support for using storytelling. These determinants are influenced by the moderators: gender, age, experience, and voluntariness of use. Figure 1 illustrates the research model.

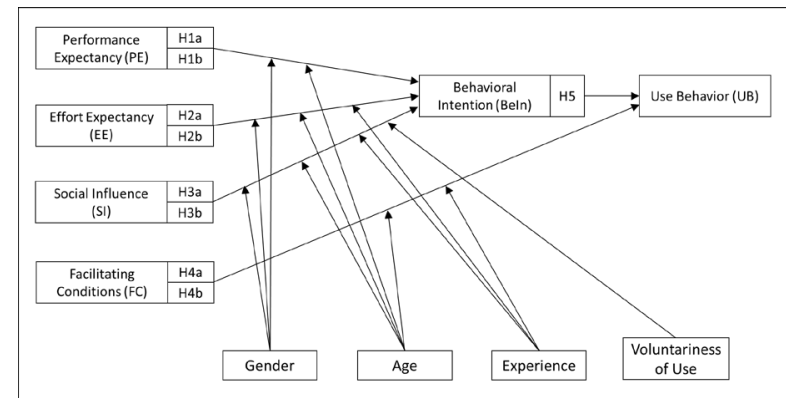

Figure 1. Research model following [40]

Deviating from Venkatesh et al. [40], the following hypothesized relationships were generated in two steps $(\mathrm{a}=$ relationship between the latent variables; $\mathrm{b}=$ moderated influence on the relationships between the latent variables). For example, hypothesis H1a und $\mathrm{H} 1 \mathrm{~b}$ are formulated as follows: "Performance expectancy is positively associated with the intention to use storytelling in business intelligence systems." and "The influence of performance expectancy on the intention to use storytelling in business intelligence systems is moderated by gender and age, with a stronger 
effect on young men.”. The remaining hypotheses can be formulated similarly.

The items were adopted from Venkatesh et al. [40] and modified by replacing the word "system" with "storytelling", as shown in Table 2. In this table, the changes compared to the original items are highlighted in italics. For some items, the respondents could add comments about their understanding of the topic to ensure comprehensibility and to support the interpretation of the results.

Table 2. Measurement items

\begin{tabular}{|c|c|}
\hline Construct & Items \\
\hline \multirow{4}{*}{$\begin{array}{l}\text { Performance } \\
\text { Expectancy } \\
\text { (PE) }\end{array}$} & I find storytelling useful for my daily work. \\
\hline & $\begin{array}{l}\text { Using storytelling enables me to accomplish } \\
\text { tasks more quickly. }\end{array}$ \\
\hline & $\begin{array}{l}\text { Using storytelling has a positive effect on my } \\
\text { productivity. }\end{array}$ \\
\hline & $\begin{array}{l}\text { If I use storytelling, I will increase my chances of } \\
\text { getting a raise. }\end{array}$ \\
\hline \multirow{4}{*}{$\begin{array}{l}\text { Effort } \\
\text { Expectancy } \\
\text { (EE) }\end{array}$} & $\begin{array}{l}\text { My interaction with storytelling is clear and } \\
\text { understandable. }\end{array}$ \\
\hline & $\begin{array}{l}\text { It is easy for me to become skillful at using } \\
\text { storytelling. }\end{array}$ \\
\hline & I find storytelling easy to use. \\
\hline & Learning to operate storytelling is easy for me. \\
\hline \multirow{4}{*}{$\begin{array}{l}\text { Social } \\
\text { Influence } \\
\text { (SI) }\end{array}$} & $\begin{array}{l}\text { People who influence my behavior think that I } \\
\text { should use storytelling. }\end{array}$ \\
\hline & $\begin{array}{l}\text { People who are important to me think that I } \\
\text { should use storytelling. }\end{array}$ \\
\hline & $\begin{array}{l}\text { The senior management of this business has } \\
\text { been helpful in the use of storytelling. }\end{array}$ \\
\hline & $\begin{array}{l}\text { In general, the organization has supported the } \\
\text { use of storytelling. }\end{array}$ \\
\hline \multirow{3}{*}{$\begin{array}{l}\text { Facilitating } \\
\text { Conditions } \\
\text { (FC) }\end{array}$} & $\begin{array}{l}\text { I have the resources necessary to use } \\
\text { storytelling. }\end{array}$ \\
\hline & $\begin{array}{l}\text { I have the knowledge necessary to use } \\
\text { storytelling. }\end{array}$ \\
\hline & $\begin{array}{l}\text { A specific person (or group) is available for } \\
\text { assistance with storytelling difficulties. }\end{array}$ \\
\hline \multirow{3}{*}{$\begin{array}{l}\text { Behavioral } \\
\text { Intention } \\
\text { (Beln) }\end{array}$} & I intend to use storytelling the next months. \\
\hline & $\begin{array}{l}\text { I predict I would use storytelling in the next } \\
\text { months. }\end{array}$ \\
\hline & I plan to use storytelling in the next months. \\
\hline $\begin{array}{l}\text { Use Behavior } \\
\text { (UB) }\end{array}$ & $\begin{array}{l}\text { Please indicate the percentage of your working } \\
\text { week that you use the function storytelling in a BI } \\
\text { system: }(0 \% / 20 \% / 40 \% / 60 \% / 80 \% / 100 \%)\end{array}$ \\
\hline Age & How old are you? \\
\hline Gender & What gender do you belong to? \\
\hline Experience & $\begin{array}{l}\text { What experience do you have with the usage of } \\
\text { storytelling in BI systems? } \\
\text { (0 months / up to } 3 \text { months / up to } 6 \text { months / } \\
\text { up to } 12 \text { months / more than } 12 \text { months) }\end{array}$ \\
\hline $\begin{array}{l}\text { Voluntariness } \\
\text { of Use }\end{array}$ & $\begin{array}{l}\text { How do you use storytelling? (7-point Likert scale } \\
\text { from nonvoluntary to completely voluntary) }\end{array}$ \\
\hline
\end{tabular}

All items were rated on a 7-point Likert scale (from strongly disagree to strongly agree) [40]. One original item has been removed due to its irrelevance in our context: "The system is not compatible with other systems I use." Storytelling is a function that is implemented and used in a BI tool, therefore no compatibility with other systems is necessary. Due to the lack of access to log data of the BI tools, the item about the usage was measured with an interval scale to guarantee a correct measurement. We added a question about the usage of the functions reporting and dashboarding to enable a comparison and understanding of the results for storytelling. In reference to Venkatesh et al. [40] the moderators were measured with single items. The moderator experience in storytelling was, in contrast to Venkatesh et al. [40], extended from a 3-point scale with small, medium, large level of experience to a 5-point scale with a more precise description of time periods to ensure the correct understanding by the participants.

For the questions on age and gender the option "not applicable" was given. In addition, several other statistical, demographic, and BI-related questions were asked to capture (personal and professional) characteristics of BI users (e.g. company size, industry, department, nationality, headquarters location, BI tool used, user role, experience in BI, etc.).

\subsection{Data Collection}

For data collection, an online questionnaire survey was chosen as form of quantitative research. The initial survey was revised in a pre-test by experts from research and practice in order to determine comprehensibility, structure, and completeness as well as the expected duration to complete the survey. The study was limited to BI users who we contacted online via the business social media platforms Xing and LinkedIn as well as via e-mail through our network of experts in this area. The survey was conducted in German and in English in order to exclude any falsification of the answers due to language understanding problems and also to consider internationally comparable results. We intentionally addressed with the survey various BI user roles to ensure a reliable and valid sample. We defined the roles for the study as follows: information consumer, analyst, specialist, administrator, database developer, and (software) developer. All roles represent potential users of the storytelling feature. We were interested in which role(s) and how often per role (on a 5-point Likert scale from never to always) the participants use the BI tool. Therefore, we have included this question in the survey as well. To better understand if the participants in the survey belong to the target group, we also asked for the experience with BI tools in years, their department within the organization as well as the concrete BI tool(s) they use.

The survey was conducted from the 12 December 2019 to the 11 February 2020 and reached 195 participants. After data cleansing with discarding 
incomplete and inconsistent questionnaires, 113 questionnaires were available as data set for the analysis. In total 69 questionnaires were answered in German and 44 in English.

\section{Results}

This section presents the results starting with descriptive statistics. After verifying the factor loadings, reliability, and validity of the measurement models, the results of the hypothesis testing are presented.

\subsection{Descriptive Statistics}

SPSS software was used for data cleansing and for descriptive statistics. About two thirds of the participants were $(72 \%)$ male and one third were (27\%) female. About $80 \%$ were between 22 and 42 years old and almost half of the participants were younger than 32 years $(46 \%)$. While $28 \%$ of the respondents had up to two years of experience in BI, the figure is $22 \%$ for two to five years and $11 \%$ for five to seven years. A total of $37 \%$ had even more than 7 years of experience in $\mathrm{BI}$.

A total of $61 \%$ of the participants had German nationality, followed by India, the USA, and the UK. Similarly, the results about the industry and department in which the respondents work were broadly scattered. The most frequent industries were telecommunications, IT and software industry, consulting, and mechanical and metal industry. About $30 \%$ of the participants work in the IT department (30\%), followed by controlling $(22 \%)$, sales and marketing $(20 \%)$, and management (18\%).

In terms of the BI tool used by respondents, a wide range was named, but the three most popular tools were Microsoft PowerBI (25,2\%), Tableau (18,6\%), and IBM Cognos Analytics (15,5\%).

About two thirds of the participants used storytelling at the time of the survey. In comparison to the BI features reporting $(97,3 \%)$ and dashboarding (92,9\%), the use of storytelling was considerably lower.

Table 3. User roles and their use of storytelling

\begin{tabular}{|l|c|}
\hline User Role & Usage more than 20 \% per week \\
\hline Information Consumer & $\mathbf{7 5 , 4} \%$ \\
\hline Analyst & $80,3 \%$ \\
\hline Specialist & $78,8 \%$ \\
\hline Administrator & $71,1 \%$ \\
\hline Database Developer & $75,0 \%$ \\
\hline (Software) Developer & $82,8 \%$ \\
\hline
\end{tabular}

An analysis of the percentage of weekly working time spent on storytelling shows that the use of storytelling is independent of the user role (cf. Table 3).
On average, around $70 \%$ to $80 \%$ of the BI users spend more than $20 \%$ of their total weekly working time in the respective role on storytelling.

The question about the experience with storytelling resulted in $35 \%$ participants who had no experience at all, but $30 \%$ who already had more than 12 months of experience with storytelling in BI. The attendees without any experience in storytelling were asked to state their expectations or ideas about the use of storytelling in BI in the survey.

\subsection{Measurement Model}

The model has been tested using the structural equation modeling (SEM)-technique Partial Least Squares (PLS), which has fewer demands in sample size and scales and no distributional assumptions in contrast to other approaches like LISREL or AMOS [17, 40]. The software SmartPLS 3 [30] was used for the analysis. The measurement model was tested for validity and reliability criteria in order to evaluate whether their quality is sufficient to represent the constructs. In reference to Hair et al. [17], the following criteria were tested.

All items had significant and strong loading values $(>0,7)$ [17], except FC03 with less than 0,7. However, we considered it acceptable because the value is very close to 0,7 and Hair et al. [17] recommend removing items with loadings lower than 0,4. The coefficients Cronbach's alpha and composite reliability (CR) indicate the internal consistency, which were both tested as fulfilled with values greater than 0,7 [17] as shown in Table 4.

Table 4. Cronbach's alpha, composite reliability and average variance extracted

\begin{tabular}{|l|c|c|c|}
\hline Variable & $\boldsymbol{\alpha}$ & CR & AVE \\
\hline Beln & 0,966 & 0,978 & 0,937 \\
\hline PE & 0,860 & 0,906 & 0,708 \\
\hline EE & 0,867 & 0,909 & 0,714 \\
\hline SI & 0,846 & 0,896 & 0,683 \\
\hline FC & 0,644 & 0,809 & 0,587 \\
\hline
\end{tabular}

But the very high value 0,9775 of $C R$ of the construct BeIn supposes redundant items and thus a possibly invalid measurement of the construct. In addition, the Cronbach's alpha of the construct FC is slightly below the limit. Nevertheless, an overall assessment of the criteria allows the assumption of consistent results and a given internal consistency.

The convergent validity of the measurement model was tested with average variance extracted (AVE). All constructs have AVE values above 0.5, which means, that every construct explains more than half of the variance of its indicators [17]. The discriminant validity 
indicates the extent to which a construct differs from other constructs [17] and was measured by the heterotrait-monotrait ratio of correlations (HTMT). All construct combinations were under 0,85 and result in a given discriminant validity. Additionally, the $95 \%$ confidence interval was built via bootstrapping with 5000 subsamples to test the significance. At this point, the combination FC-EE had to be noticed as critical value. Still, the discriminant validity was considered as given. In accordance with Venkatesh et al. [40], all constructs used in the analysis are valid and reliable.

\subsection{Structural Model}

For the evaluation of the structural model, the collinearity between the constructs was measured by the variance inflation factor (VIF) [17]. All values were below 5 and fulfilled the criterion of collinearity. Based on the acceptable validity and reliability, the hypotheses were tested by estimating the path coefficients with PLS. The path coefficients indicate the strengths of the relationships between the latent variables [17], in our case they indicated positive relationships between the constructs. In order to assess the significance, the pvalue was generated by bootstrapping with 5000 subsamples.

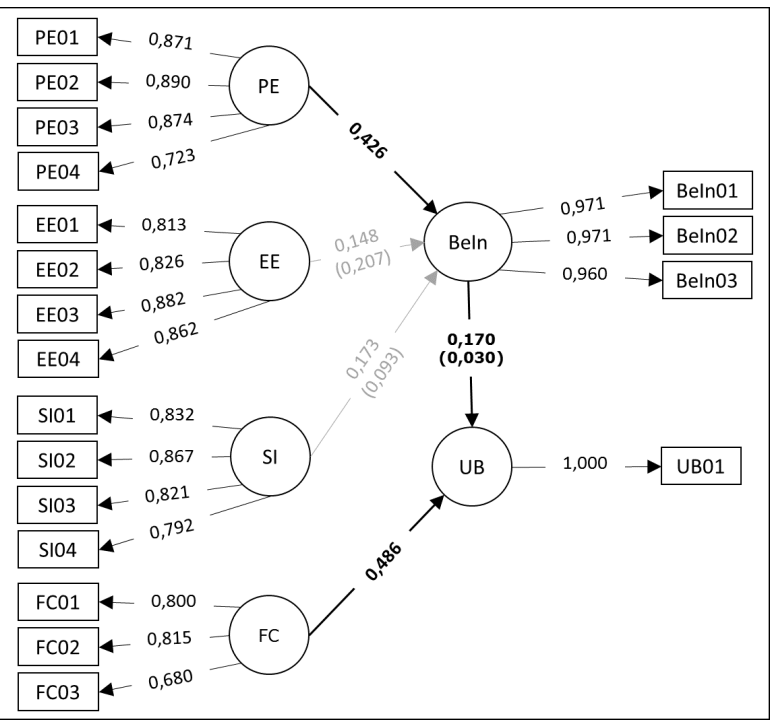

Figure 2. Results of PLS estimation in the path model with $\mathrm{p}<0,05$

Figure 2 shows the three significant positive relationships between $\mathrm{PE}$ and BeIn, BeIn and UB as well as FC and UB, supporting the hypotheses H1a, $\mathrm{H} 4 \mathrm{a}$, and $\mathrm{H} 5$. In contrast, $\mathrm{H} 2 \mathrm{a}$ and $\mathrm{H} 3 \mathrm{a}$ could not be confirmed. The figure also includes the indicators of the constructs and their factor loadings, which are all significant, and the path coefficients of the relationships between the constructs, which are extended by the pvalues.

The $\mathrm{R}^{2}$ is an indicator of the quality of the structural model by representing the variance explained by the independent variables [17]. The variance of variable BeIn was explained by the other constructs with $41 \%$ (adjusted $\mathrm{R}^{2}: 0,3985$ ). The variable $\mathrm{UB}$ had an $\mathrm{R}^{2}$ of $33 \%$ (adjusted $\mathrm{R}^{2}$ : 0,3247 ). In comparison to the theory [40], the $\mathrm{R}^{2}$ is considerably lower $(70 \% ; 50 \%)$. However, Chin [6] regards a value of $33 \%$ as moderate. Therefore, the $\mathrm{R}^{2}$ was considered as fulfilled in this case.

Furthermore, the $\mathrm{f}^{2}$ effect size was tested to see which constructs contributed to the $\mathrm{R}^{2}$ values. According to Hair et al. [17], the guidelines $\geq 0,02$, $\geq 0,15$ and $\geq 0,35$ represent small, medium, and large effect sizes. Therefore, a small effect of the constructs EE and SI on BeIn and the construct BeIn on UB could be observed. The constructs PE and FC had a medium effect size on the constructs BeIn and UB. The predictive relevance of the model was tested with the measure $\mathrm{Q}^{2}$. According to Hair et al. [17], the measurement was calculated by using the blindfolding procedure with an omission distance $\mathrm{D}=7$. The results of $\mathrm{Q}^{2}=0,3778$ for $\mathrm{BeIn}$ and $\mathrm{Q}^{2}=0,3101$ for $\mathrm{UB}$ indicate the relevance of the model. For detailed information about the impact of the constructs on the $\mathrm{Q}^{2}$ value, the $\mathrm{q}^{2}$ effect size was tested. Using the same guidelines as for the $\mathrm{f}^{2}$ effect size, the constructs $\mathrm{EE}$ and SI exhibited a small and PE a medium predictive relevance on BeIN. FC had medium predictive relevance on the construct UB.

The hypotheses about the effects of the moderators were tested by a multigroup analysis. This method requires categorial variables to split into two or more groups and estimates the model for each group to identify significant differences between der groups [17]. The results were contrary to the results of Venkatesh et al. [40], as all hypotheses H1b, H2b, H3b, and H4b were rejected. However, the following significant effects could be identified: Gender has a significant effect on the relationship between $\mathrm{FC}$ and $\mathrm{UB}$, such that the effect was stronger for men than for women. Age comprised a moderating effect on the influence of BeIn on UB so that the effect was stronger for older users. Experience moderated the relationship between PE and BeIn and between BeIn and UB. Whereby the effect was significantly stronger for users with more experience in storytelling. Additionally, these relationships were significantly moderated by voluntariness of use, such that the effect was stronger for users with a voluntary usage. Conversely, a mandatory usage had a higher effect on the relationship between FC and UB. 


\section{Discussion}

Based on the UTAUT model the constructs of the BI users' behavioral intention (acceptance) and use behavior were tested. The evaluation confirmed behavioral intention and facilitating conditions as constructs for the use behavior as well as performance expectancy as a construct of behavioral intention. The results are based on a representative sample with regards to participants in various BI user roles, in various departments in their organization and across several countries.

It seems reasonable that the behavioral intention has a significant positive effect on the use. If the use of storytelling should be increased, the acceptance and intention to use the feature should be enhanced. The moderating effect on this relationship is increased for older male BI users with much experience and voluntary use.

The positive significant influence of performance expectancy confirms, that BI users see an increase in performance through the use of storytelling. Consequently, this construct should be addressed, if the behavioral intention and acceptance of storytelling needs to be improved. BI users expect or see a benefit for their daily work, a fast completion of their tasks and a positive effect on their productivity with the justification for a salary increase by using storytelling. The open questions in the survey allowed an insight into how the participants understand productivity and the reasons for a salary increase. We assume that storytelling influences the productivity mainly through a more efficient way of working $[14,36,46]$ as well as faster and more effective analysis and presentations (e.g. $[15,20,34,44])$. This implicates that the participants see the benefits for their productivity in time saving and easily accessible results that foster the understanding. Furthermore, these benefits were noticed as reasons for a salary increase by the participants. Lastly, the effect of the performance on behavioral intention and acceptance is mainly stronger for BI users with more experience and with a voluntary use.

The strongest significant effect could be identified for the construct facilitating conditions on the use behavior. Apparently, the use of storytelling can be increased by organizational and technical availability and support [11, 27, 44]. Especially assistance and trainings were noticed by the participants.

The constructs effort expectancy and social influence were identified as not significant. It is surprising that usability has no influence on the use of storytelling by the BI users. A potential explanation could be that BI tools are already perceived as rather user-friendly, so users may not expect or not feel the need for an additional improvement of such aspects (compared to the aforementioned arguments of performance and productivity). Another assumption would be that some respondents had difficulties in assessing the usability due to their low experience in storytelling. Nevertheless, storytelling seems to be a feature that is used not only occasionally (69\% BI users used storytelling more than $20 \%$ of their weekly working time) but intensively.

As the confirmed effects are significantly influenced by the voluntary nature of use, it is not surprising that social influence also has a minor impact on the acceptance of the storytelling feature in BI tools. We expect no social pressure to use storytelling if someone does his/her work well and uses alternatives to storytelling, such as dashboards or reports.

The study results bridge the gap between research and practice and support the importance of focusing on storytelling in BI [18]. It has been shown that use of the storytelling feature in BI tools is mainly driven by facilitating conditions, so that organizational and technical availability, as well as support through best practices and training, prove to be extremely important in practice. Concrete advise how the enhancement of the facilitating conditions can be achieved might be derived from our morphological box. For example, resources provided and knowledge (items for facilitating conditions) should enable all intention options (a dimension in the morphological box) for storytelling.

\section{Conclusion and Further Research}

In the paper at hand, we have designed a morphological box for storytelling in BI, based on a literature review, and presented a quantitative study to test the use and acceptance of the storytelling feature in BI tools. As we have shown, there is no "standard" for the use of storytelling in BI, but many different ways to do so. From a research point of view, the morphological box presents characteristics of storytelling in BI, which are likely to become more important in the upcoming years. It provides valuable insights into the current practice and a possible framework for its categorization and analysis. Scientists and organizational decisionmakers are informed about the state of the art in storytelling for BI and are given an overview of the various ways in which it can be used.

An overall evaluation of the morphological box based on a comparison with practical literature and specific BI tools in practice will be subject to future work. We plan not only to analyze specific BI tools but also to classify other tools for storytelling in BI according to the morphological box. This also includes adding further attributes and/or dimensions to the morphological box, referring to general characteristics of BI, but also relevant for storytelling in BI. For 
example, the distinction between internal and/or external use as well as further BI user roles. On the basis of the revised artifact, we also want to investigate which application scenarios are particularly suitable for storytelling.

When interpreting the empirical results, the limitations of the underlying empirical analysis should be considered. The survey is limited with regards to the measurement of the variable use behavior. Contrary to the theoretical model [40], which measured the system usage with log data, we measured the use of storytelling by an interval scale, leading to a lower accuracy in comparison to the theory. Besides, some results of quality criteria show critical values that require control procedures. The variable facilitating conditions has a critical Cronbach's alpha. Nevertheless, the other criteria show acceptable values for keeping the variable. The high value of composite reliability $(0,9775)$ assumes redundant indicators of the variable behavioral intention. Unfortunately, due to a lack of research, we were not able to compare these results with similar studies. Although the $\mathrm{R}^{2}$ of the model can be classified as acceptable, the values of $41 \%$ and $33 \%$ indicate that more than half of the variance is caused by unknown factors. This shows that the study is limited regarding the determinants of UTAUT and should investigate in future research further potential constructs with impact on behavioral intention and use behavior. Also, the contrary results of the moderator effects compared to the theory of Venkatesh et al. [40] need to be checked in future research. There are limitations in the sample size, which results in small sizes in the distribution multigroup analysis, as well as in the choice of the approach and in the formulated hypotheses in reference to the theory of Venkatesh et al [40]. We recommend future research in a separate study about the moderators, where hypotheses, approach etc. are checked for compatibility.

Despite the above-mentioned limitations, we are confident that we could contribute with our research new insights to practice and to the scientific community by highlighting various facets of storytelling for BI and thus showing a new way to think about BI.

\section{References}

[1] Baier, L., C. Bange, A. Baumhecker, et al., BI Trend Monitor 2020: BARC Research Study, 2019.

[2] Boy, J., F. Detienne, and J.D. Fekete, "Storytelling in Information Visualizations: Does it Engage Users to Explore Data?", Proceedings of the 33rd Annual ACM Conference on Human Factors in Computing Systems (CHI'15), (2015), 1449-1458.

[3] Brown, J., S. Denning, K. Groh, and L. Prusak, Storytelling in Organizations: Why Storytelling Is Transforming 21st Century Organizations and Management, Elsevier
Butterworth-Heinemann, Burlington, Oxford, 2005.

[4] Chen, Q., Z. Li, T.C. Pong, and H. Qu, "Designing Narrative Slideshows for Learning Analytics", IEEE Pacific Visualization Symposium, IEEE (2019), 237-246.

[5] Chen, S., J. Li, G. Andrienko, et al., "Supporting Story Synthesis: Bridging the Gap between Visual Analytics and Storytelling", IEEE Transactions on Visualization and Computer Graphics 26(7), 2020, pp. 2499-2516.

[6] Chin, W.W., "The Partial Least Squares Approach to Structural Equation Modeling", In G.A. Marcoulides, ed., Modern Methods for Business Research. Lawrence Erlbaum, Mahwah, New Jersey, London, 1998, 295-336.

[7] Dale, E., Audiovisual Methods in Teaching, Dryden Press, New York, 1969.

[8] Damodaran, A., Narrative and Numbers: The Value of Stories in Business, Columbia University Press, New York, Chichester, 2017.

[9] Dinter, B., and A. Lorenz, "Social Business Intelligence: A Literature Review and Research Agenda", Proceedings of the 33rd International Conference on Information Systems, ICIS 2012, (2012), 1-12.

[10] Eccles, R., T. Kapler, R. Harper, and W. Wright, "Stories in GeoTime", Information Visualization 7(1), 2008, pp. 3-17.

[11] Echeverria, V., R. Martinez-Maldonaldo, S. Buckingham Shum, K. Chiluiza, R. Granda, and C. Conati, "Exploratory versus Explanatory Visual Learning Analytics: Driving Teachers' Attention through Educational Data Storytelling", Journal of Learning Analytics 5(3), 2018, pp. 72-97.

[12] Elias, M., M.-A. Aufare, and A. Bezerianos, "Storytelling in Visual Analytics Tools for Business Intelligence", In P. Kotzé, G. Marsden, G. Lindgaard, J. Wesson and M. Winckler, eds., Human-Computer Interaction, INTERACT 2013: 14th IFIP TC 13 International Conference, Proceedings. Springer-Verlag, Berlin, Heidelberg, 2013, 280-297.

[13] Faddoul, G., and S. Chatterjee, "The Virtual Diabetician: A Prototype for a Virtual Avatar for Diabetes Treatment Using Persuasion Through Storytelling", Proceedings of the 25th Americas Conference on Information Systems, AMCIS 2019, (2019), 1-10.

[14] Figueiras, A., "Narrative Visualization: A Case Study of How to Incorporate Narrative Elements in Existing Visualizations", Proceedings of the 18th International Conference on Information Visualisation, IEEE (2014), 46-52.

[15] Gershon, N., and W. Page, "What Storytelling Can Do for Information Visualization", Communications of the ACM 44(8), 2001, pp. 31-37.

[16] Gutiérrez, A., and C.B. Pérez, "Data visualization process through storytelling technique in Business Intelligence", Avances en Interacción Humano-Computadora 1(1), 2016, pp. 49-52.

[17] Hair, J.F., B.J. Babin, R.E. Anderson, and W.C. Black, Multivariate Data Analysis, Cengage Leraning EMEA, London, 2018.

[18] Herschel, R.T., and N. Clements, "The Importance of Storytelling in Business Intelligence", International Journal of Business Intelligence Research 8(1), 2017, pp. 26-39. 
[19] Howson, C., J. Richardson, R. Sallam, and A. Kronz, "Magic Quadrant for Analytics and Business Intelligence Platforms", 2019.

https://www.gartner.com/doc/reprints?id=1$68720 \mathrm{FP} \& \mathrm{ct}=190213 \& \mathrm{st}=\mathrm{sb}$

[20] Kosara, R., and J. MacKinlay, "Storytelling: The Next Step for Visualization", Computer 46(5), 2013, pp. $44-50$.

[21] Lee, B., R.H. Kazi, and G. Smith, "SketchStory: Telling More Engaging Stories with Data through Freeform Sketching", IEEE Transactions on Visualization and Computer Graphics 19(12), 2013, pp. 2416-2425.

[22] Lee, B., N.H. Riche, P. Isenberg, and S. Carpendale, "More Than Telling a Story: Transforming Data into Visually Shared Stories", IEEE Computer Graphics and Applications 35(5), 2015, pp. 84-90.

[23] Lin, C., "Data Driven Product Management", IEEE Engineering Management Review 46(1), 2018, pp. 16-18.

[24] Lugmayr, A., E. Sutinen, J. Suhonen, C.I. Sedano, H. Hlavacs, and C.S. Montero, "Serious storytelling - a first definition and review", Multimedia Tools and Applications 76(14), 2017, pp. 15707-15733.

[25] Ma, K.L., I. Liao, J. Frazier, H. Hauser, and H.N. Kostis, "Scientific Storytelling using Visualization", IEEE Computer Graphics and Applications 32(1), 2012, pp. $12-19$.

[26] Marjanovic, O., "Using Collaborative Visual Analytics for Innovative Industry-inspired Learning Activities", Proceedings of the 26th Australasian Conference on Information Systems, ACIS 2015, (2015), 1-12.

[27] Marjanovic, O., "Empowering Business Users to Explore Visual Data Through Boundary objects and Storytelling", Proceedings of the 49th Hawaii International Conference on System Sciences, HICSS 2016, IEEE (2016), 5032-5041.

[28] Moretti, M., F. De Chiara, and M. Napolitano, "Beyond Transparency: making the Italian Public Administration more accessible through data storytelling", Proceedings of the 22nd International Conference Information Visualisation, (2018), 247-250.

[29] Pribisalić, M., I. Jugo, and S. Martinčić-Ipšić, "Selecting a Business Intelligence Solution that is Fit for Business Requirements", Proceedings of the 32nd Bled eConference, BLED 2019, (2019), 443-465.

[30] Ringle, C.M., S. Wende, and J.-M. Becker, "SmartPLS 3", SmartPLS, 2015. http://www.smartpls.com

[31] Rodríguez, M.T., S. Nunes, and T. Devezas, "Telling Stories with Data Visualization", Proceedings of the 2015 Workshop on Narrative and Hypertext, NHT 2015, (2015), 7-11.

[32] Saundage, D., J.L. Cybulski, S. Keller, and L. Dharmasena, "Teaching Data Analysis with Interactive Visual Narratives", Journal of Information Systems Education 27(4), 2016, pp. 233-248.

[33] Schiller, S., "Storytelling with Tableau: A Hands-on Workshop", Midwest Association for Information
Systems (MWAIS) Conference, (2017), 1-3.

[34] Schwabe, G., A. Richter, and E. Wende, "Special issue on storytelling and information systems", Information Systems Journal 29(6), 2019, pp. 1122-1125.

[35] Segel, E., and J. Heer, "Narrative Visualization: Telling Stories with Data", IEEE Transactions on Visualization and Computer Graphics 16(6), 2010, pp. 1139-1148.

[36] Seyser, D., and M. Zeiller, "Scrollytelling-An Analysis of Visual Storytelling in Online Journalism", Proceedings of the 22nd International Conference Information Visualisation, IEEE (2018), 401-406.

[37] Stolper, C.D., B. Lee, N.H. Riche, and J. Stasko, "DataDriven Storytelling Techniques: Analysis of a Curated Collection of Visual Stories", In N.H. Riche, C. Hurter, N. Diakopoulos and S. Carpendale, eds., Data-Driven Storytelling. Taylor \& Francis Ltd., Boca Raton, New York, Milton, 2018, 85-105.

[38] Turban, E., R.E. Sharda, and D. Delen, Decision Support and Business Intelligence Systems, Prentice Hall, New Jersey, 2011.

[39] Veel, K., "Make data sing: The automation of storytelling", Big Data and Society 5(1), 2018, pp. 1-8.

[40] Venkatesh, V., M.G. Morris, G.B. Davis, and F.D. Davis, "User Acceptance of Information Technology: Toward a Unified View”, MIS Quarterly 27(3), 2003, pp. 425-478.

[41] Wang, D., and R. Santhanam, "Visual Storytelling: Impact of Data visualization on citizens' health behaviors", Proceedings of the 10th Midwest Association for Information Systems Conference, MWAIS 2015, (2015), 1-5.

[42] Watson, H.J., "Data Visualization, Data Interpreters, and Storytelling”, Business Intelligence Journal 22(1), 2017, pp. 5-11.

[43] Webster, J., and R.T. Watson, "Analyzing the Past to Prepare for the Future: Writing a Literature Review.", MIS Quarterly 26(2), 2002, pp. xiii-xxiii.

[44] Weissenfeld, K., O. Abramova, and H. Krasnova, "Understanding Storytelling in the Context of Information Systems", Proceedings of the 23rd Americas Conference on Information Systems, AMCIS 2017, (2017), 1-10.

[45] Williams, M.D., N.P. Rana, and Y.K. Dwivedi, "The unified theory of acceptance and use of technology (UTAUT): a literature review", Journal of Enterprise Information Management 28(3), 2015, pp. 443-448.

[46] Wojtkowski, W., and W.G. Wojtkowski, "Storytelling: its role in information visualization", Proceedings of European Systems Science Congress, (2002), 1-5.

[47] Zhao, Y., T. Blount, L. Koesten, and E. Simperl, "Structured and Uncertainty-Aware Data Storytelling", CHI '19 Workshop on HCI for Accurate, Impartial and Transparent Journalism: Challenges and Solutions, (2019), 1-5.

[48] Zwicky, F., Discovery, Invention, Research Through the Morphological Approach, MacMillian, New York, 1969. 